\title{
Protestforschung zwischen allen Stühlen
}

\section{Ein Versuch über die Sozialfigur des „Protestforschers“}

Peter Ullrich

\section{1| Protestforschung im politischen Auftrag? ${ }^{1}$}

Es gab in letzten Jahren einige heftige Angriffe auf die Protest- und Bewegungsforschung. In Flugblättern, bei Veranstaltungen insbesondere in Frankfurt, auf dem Portal „German Foreign Policy“ und in der Tageszeitung, ,junge Welt“ wurde der Protestforschung nicht weniger vorgehalten als „repressive ... Gegnerkunde“ (Heinelt 2017) zu sein. Mit ihren Untersuchungen mache sich die „konterrevolutionäre Forschung“2 zum „Gehilfen des Staatsschutzes“, so der Journalist Peer Heinelt (2017). Das greift Bedenken auf, die in der Linken gegenüber der Protestforschung immer wieder artikuliert wurden (vgl. Mohr/Rübner 2010). Einige der jüngeren Interventionen, die auf eine linke Frankfurter Gruppe und oben genannten Journalisten zurückgehen, sind von offensichtlich tiefer Unkenntnis des Forschungsfeldes geprägt, wird in diesen Texten doch alles in einen Topf geworfen: akademische Protestforschung, Devianzsoziologie, verfassungsschutznahe Extremismusforschung, Forschung zu Radikalisierungsprozessen. Mit der Hermeneutik des Verdachts wird in Projektabstracts eine politische Botschaft hineininterpretiert, die sich durch die Lektüre von tatsächlichen Veröffentlichungen mancher der Kritisierten schnell in Luft auflösen würde. Wenn man die narzisstische Kränkung über das offensive Missverstehen und den politischen Outingversuch beiseiteschiebt, bleibt jedoch ein reflexionswürdiger Kern. Denn angesichts einer fortschreitenden Pluralisierung des Akteursfeldes, das Proteste erforscht oder protestbezogenes Monitoring betreibt, wird immer deutlicher, dass die Grenzen zwischen bewegungssoziologischer Grundlagenforschung und „Protestforschung mit politischem Auftrag" (Teune/Ullrich 2018) durchaus fließend sind. ${ }^{3}$
Ich gehe im Folgenden der Frage nach, wie berechtigt der Vorwurf einer ,Gegnerkunde“ ist. Dies erfolgt durch die Analyse der verschiedenen Beziehungsmuster zwischen den „Protestforscher"innen" und ihrem Feld, die Auswirkungen auf die Art und Weise des produzierten Wissens haben können (Abschnitt 2) und durch Überlegungen zur gesellschaftlichen Nützlichkeit von Protestforschung, die in den harschen Kritiken kaum Beachtung finden (Abschnitt 3). Diese Nützlichkeit steht aber unter dem Vorbehalt der erfolgreichen Lösung forschungsethischer Herausforderungen in einem schwierigen Forschungsfeld (Abschnitt 4). Verbinden möchte ich all dies mit einem Plädoyer für Protestforschung - aber nicht irgendeine, sondern als soziologische Aufklärung für die Gesellschaft und die Bewegungen selbst.

\section{2| Beziehungsmuster: Protestforscher*in- nen und ihr Feld}

Das Verhältnis der Forschenden zu ihrem Feld ist in der Bewegungsforschung von ganz besonderer Bedeutung. Dies liegt zum ersten an den verbreiteten Forschungsmethoden und Feldzugängen. Protestforscher*innen arbeiten, als Befragende in Demonstrationen, als teilnehmende Beobachter*innen usw., häufig im engen Kontakt mit den „Objekten“ des Interesses, mit denen sie oft über viele Jahre im Austausch stehen. Sie unterscheiden sich hierin beispielsweise von der Meinungsforschung und deren unpersönlichem Verhältnis zu den Befragten oder von einer Diskursforschung, die ausschließlich aus der Distanz beobachtet. Zum zweiten liegt es an der (potenziellen) Brisanz der Inhalte. Die Beziehung der Forschenden zum Feld ist aufgrund ihrer politischen Relevanz und vielfältigen damit verbundenen Interessen eine sensible, beobachtete, bewusst reflektierte und immer wieder diskutierte. 
Die Entscheidung von Aktivist"innen über die Teilnahme an Projekten der Protestforschung findet daher nicht ad hoc statt, sondern in einem diskursiv vorstrukturierten Raum. Eine wichtige Rolle darin spielt die Wahrnehmung der Praxis und Funktion von Protestforschung durch die Aktivist"innen und damit die Frage, wie die Forschenden sich zum Feld positionieren. Deren zugrundeliegende Überlegung ist so naheliegend wie überzeugend: Die Stellung zum Feld hat erwartbare Auswirkungen auf die Art des produzierten Wissens, seine Verwendungsszenarien und damit auch auf die Beforschten. Wie informiert diese Einschätzung der Forschung durch Aktivist"innen jedoch erfolgt, ist ebenso fraglich wie der genaue Charakter der unterstellten Auswirkungen.

Die real vorhandenen Beziehungsmuster lassen sich entlang zweier Dimensionen beschreiben. Differenzierend ist erstens die Stärke der Positioniertheit, also die Frage, ob es sich beim Feld für die Forschenden um einen ,gewöhnlichen', mehr oder weniger austauschbaren Gegenstand wissenschaftlichen Interesses handelt, oder ob stattdessen eine spezifizierbare (normative, affektive, institutionelle etc.) Positioniertheit zum Feld vorliegt (Positioniertheitsdimension). Zum zweiten ist nach dem konkreten Inhalt etwaiger Positioniertheit zu fragen, also insbesondere, ob die explizit positionierte Beziehung eher affirmativen oder kritischen Charakters ist (Bewertungsdimension). In einem durch diese Dimensionen konstituierten Feld lassen sich die meines Erachtens prägenden Grundtypen von Protestforscher"innen sowie einige Grenzbereiche darstellen (vgl. Abb. 1). Die Typologie zielt - von der realen Vielfalt abstrahierend - auf Idealtypen, geordnet entlang der Frage, wie sich ihr Verhältnis zum eigenen Gegenstand gestaltet, der oft mehr als nur ein Forschungsgegenstand ist.

Abbildung 1: Dimensionen des Feldes und Typen von Protestforscher*innen

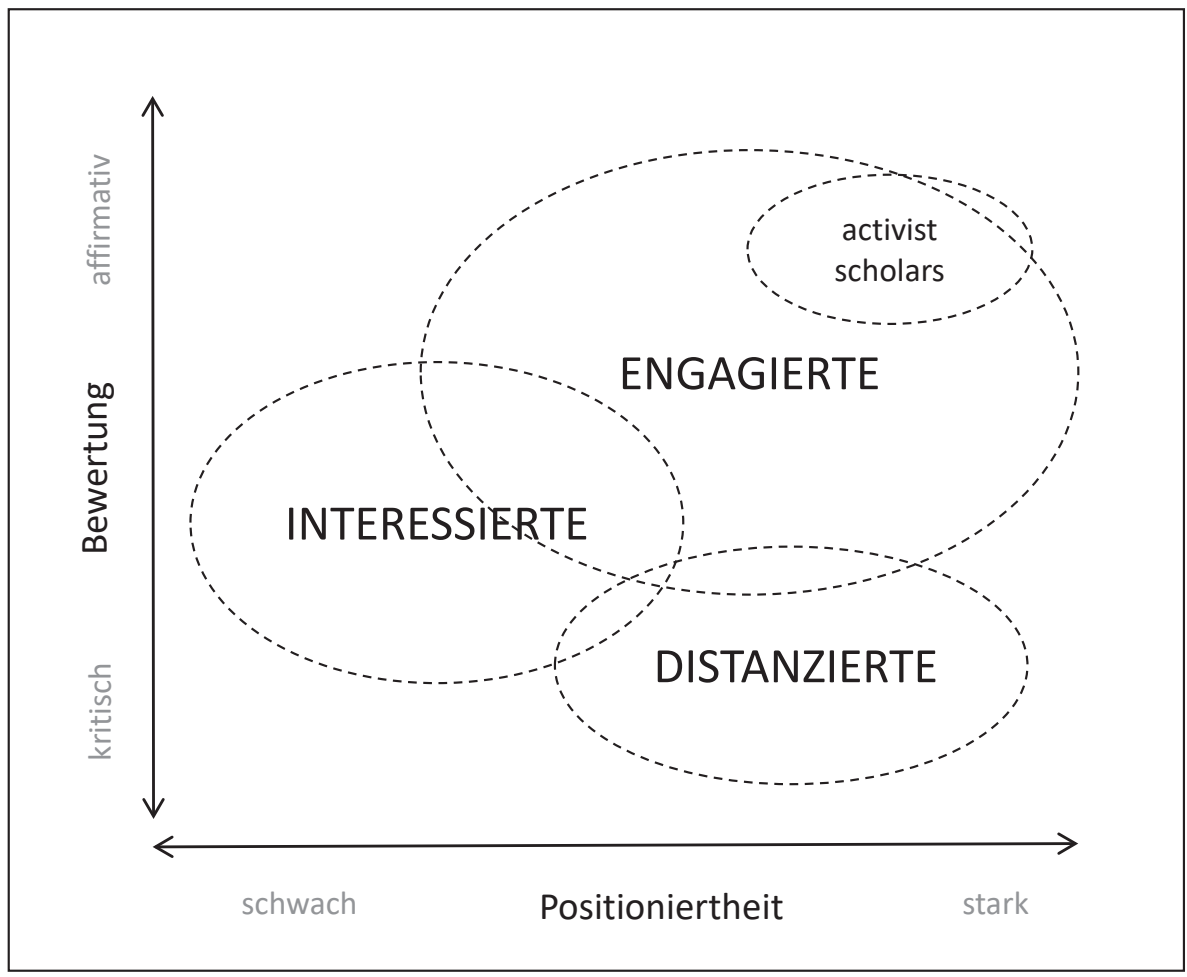




\section{Typ 1: Die Interessierten}

Ein erheblicher Teil der Forscher*innen widmet sich sozialen Bewegungen und Protesten, weil es sich um Phänomene grundsätzlicher und zudem wachsender Bedeutung handelt, zu denen es einen Bedarf an Expertise und damit ein gewisses - überschaubares - Angebot an akademischen Stellen gibt. So wie die Strukturbedingungen bewegungsförmigen Handelns auf Dauer gestellt sind (Neidhardt/Rucht 1993), ist es das Phänomen selbst. Dem sozialen Bedeutungsgewinn von Protesten entspricht die nachholende Ausdifferenzierung einer spezialisierten Teildisziplin ihrer Beobachtung. ${ }^{4}$ Vorangetrieben wird die Etablierung durch Medialisierungsprozesse, also die Ausrichtung nicht nur der Bewegungen, sondern auch der sie Erforschenden an Medienlogiken und medialer Nachfrage, die in den vergangenen etwa 15 Jahren zur Etablierung des „Protestforschers" als Expertentypus entscheidend beitrugen. Für die Interessierten sind Proteste ein Thema wie mehr oder weniger jedes andere, mit dem man eine akademische Laufbahn zumindest beginnen sowie etwas Reputation und Einkommen erwerben kann. ${ }^{5}$ Das Verhältnis zum Feld ist geprägt durch eine Mischung aus inhaltlichem Interesse und instrumentellen Momenten. Die Forschung der Interessierten ist selbstverständlich genauso wenig wertfrei wie alle Wissenschaft. Persönlich-politische Affinitäten oder Antipathien zu bestimmten Bewegungen sind nicht ausgeschlossen, aber sie sind nicht maßgeblich für das Verhältnis zum Forschungsfeld.

Primärer Bezugspunkt der Feldstrategien dieses Typs ist die Wissenschaft. ${ }^{6}$ Der Existenz dieses Typus von Forschenden ist es entsprechend zu verdanken, dass es in der Forschung zu sozialen Bewegungen trotz aller Neigungen und Voreingenommenheiten eine Orientierung an Fragestellungen und der Entwicklung von Theorie gibt, die als relevant für alle Bewegungen, unabhängig von ihrer politischen Färbung, gelten können. Die Probleme dieser Forschenden sind typischerweise nicht die Probleme, die Engagierte bewegen. Gelegentlich wird die hohe Politisierung des Forschungsfeldes durch den Typ der Engagierten von den Interessierten auch als Problem gesehen. Dieser Typus scheint im Kontext seiner universitären Institutionalisierung besonders in der US-amerikanischen Forschung präsent, orientiert sich häufiger an einem traditionellen Wissenschaftsverständnis und hat als präferierte Öffentlichkeit referierte Journals wie Mobilization und Social Movement Studies sowie Sektionen und Research Networks in den großen sozialwissenschaftlichen Fachgesellschaften.

\section{Typ 2: Die Engagierten}

Den zweiten Typus, im Bereich jüngerer Wissenschaftler*innen in Qualifikationsphasen wahrscheinlich auch die zahlenmäßig größte Gruppe an Forschenden, bilden die Engagierten. Sie sind als spezifisch Positionierte dadurch gekennzeichnet, dass Themeninteresse, Fragestellungen und Zugänge zur akademischen Protestforschung vornehmlich aus eigenem Engagement oder mindestens starken Affinitäten zu bestimmten Bewegungen resultieren.

Die Existenz dieses Typus ist wenig überraschend. Er führt eine lange, geschichtsphilosophisch geprägte Tradition akademischpolitischen Räsonierens über Bewegungen als Akteure, mit denen Hoffnungen auf eine andere und bessere Welt verbunden sind, fort. Diese Linie lässt sich mindestens bis zu Marx/Engels (und letztlich Hegel) zurückführen, umfasst einen großen Teil der kritischen Theorie(n), also Arbeiten unter anderem aus dem Kontext des Marxismus, der Cultural Studies, des Postkolonialismus und des Poststrukturalismus. Damit einher geht häufig ein Wissenschaftsverständnis, das auch für die Protestforschung mit dem Label „postfundamentalistisch“ zu fassen versucht wurde (Leinius et al. 2017). Ein im Grundsatz affirmatives Verhältnis zu (manchen) sozialen Bewegungen in diesem Sinne war auch prägend für wichtige Vertreter*innen der Forschungen zu den „Neuen Sozialen Bewegungen“ (NSB-Forschung), die zugleich maßgeblich für die akademische Institutionalisierung der Protestforschung war. Man denkt hier an Namen wie Alain Touraine. Die Entscheidung 
für ein solches Thema ist zudem für politisch interessierte Studierende mit sozialwissenschaftlichen Aspirationen, die mit Hoffnungen, Enttäuschungen und Widersprüchen des eigenen Engagements konfrontiert sind, eine äußert naheliegende Wahl. Entsprechend werden alle größeren Mobilisierungszyklen von einer Vielzahl entsprechender Masterarbeiten und Dissertationen begleitet. In diesem $\mathrm{Zu}$ gangsweg zum Forschungsfeld liegt auch die große Bedeutung der Fallanalysen einzelner Bewegungen oder Mobilisierungen innerhalb der Protestforschung begründet. Die persönliche Themenaffinität ist der Ausgangspunkt und erfährt erst im Wissenschaftssystem eine mehr oder weniger strikte akademische Überformung. Nicht zuletzt im Forschungsjournal (Neue) Soziale Bewegungen fand der Typus seinen Ausdruck. Das FJSB war immer auch, aber nie nur akademisch geprägt, immer zugleich getragen von Sympathie für progressive
Bewegungen und - nach und nach - auch Auseinandersetzungen mit Bewegungen, deren Ziele und Mittel klar abgelehnt wurden (Klein/ Hellmann 1994).

Ein besonderer Subtypus beziehungsweise eine extreme Ausprägung innerhalb der Engagierten, sind diejenigen, die man im Englischen als activist researchers oder activist scholars bezeichnet (Gillan/Pickerill 2012: 135). Sie vertreten in der Regel die Aufhebung der Grenze von Wissenschaft und Politik beziehungsweise bestreiten, dass diese grundsätzlich divergenten Logiken folgen (sollten), und stellen ihre Forschung in den Dienst von Gesellschaftskritik und Bewegungen (Vey 2015: 14ff.; vgl. auch Starodub in diesem Heft (Anm. d. Red.)). Forschung wird in der Tradition der Handlungsforschung häufig eher als Koproduktion von Wissen zusammen mit den Bewegungen zu realisieren versucht (vgl. beispielsweise The Autonomous Geographies Collective 2010).

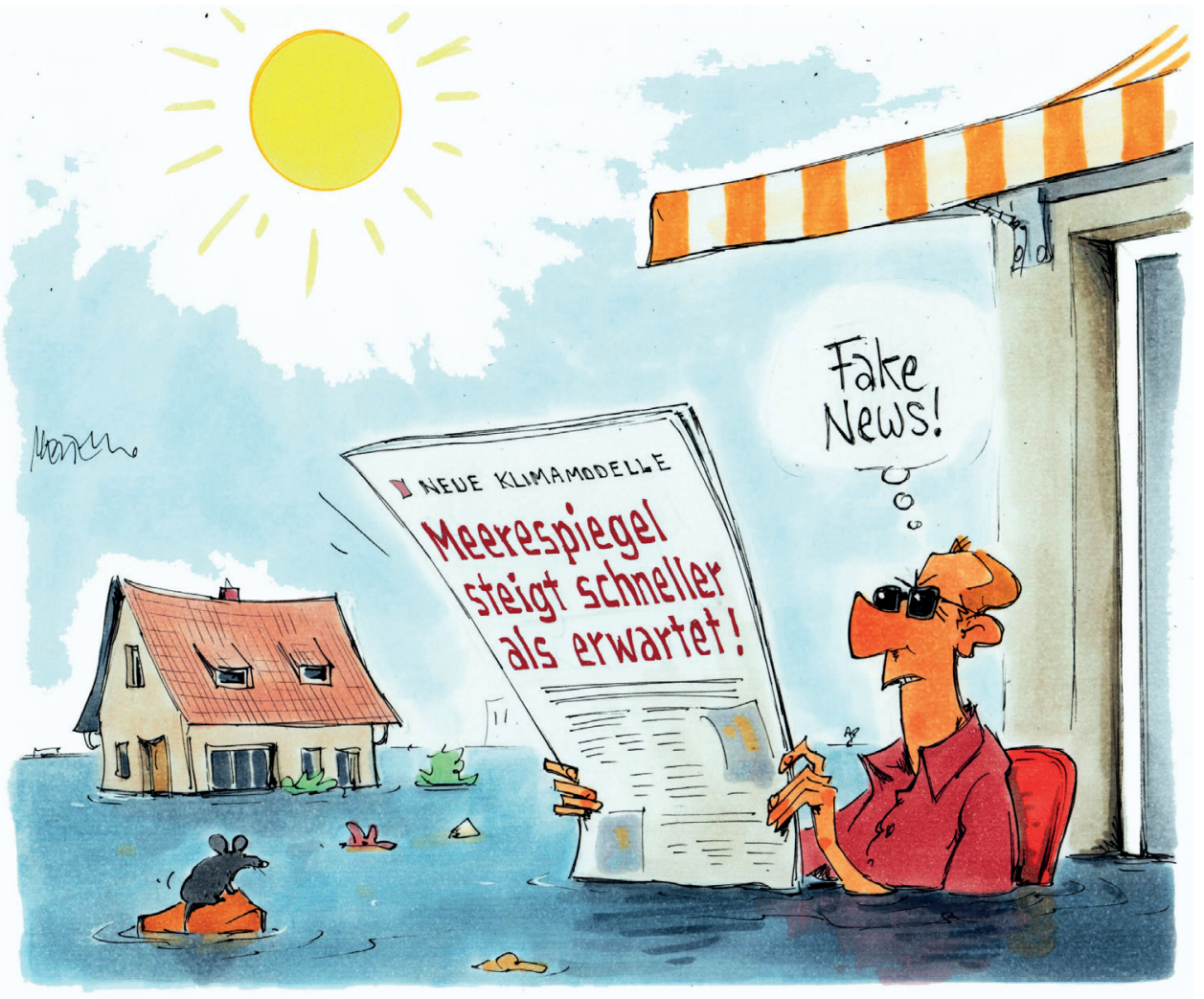


Seinen klarsten institutionellen Ausdruck und ein öffentliches Forum findet dieser Typus in der Zeitschrift „Interface - Journal for and about social movements" (Herv. PU), in dessen Peer-Review-Prozess jeweils zwei Personen die Aufsätze begutachten - ein"e in der Rolle als Wissenschaftler*in und ein"e in der Rolle als Aktivist"in -, um so wissenschaftliche Qualität und Relevanz für Bewegungen zugleich abzusichern.

\section{Typ 3: Die Distanzierten}

Der dritte Typus stellt nach meinem Dafürhalten bisher eher einen kleineren Bereich des Feldes dar, wenngleich einen mit teilweise schon realisiertem Wachstumspotenzial, welches sich aus den Anschlussmöglichkeiten an andere Forschungsfelder und sicherheitspolitische Diskurse ergibt (dazu weiter unten). Wie manche der Engagierten transportieren die Distanzierten in ihrer Beschäftigung mit sozialen Bewegungen eine stark politisch-bewertende Note, die gelegentlich vom paternalistischen Duktus ,gereifter ${ }^{\natural}$ Ex-Bewegter geprägt ist. Das eigene wissenschaftliche Schaffen mutet dann unter Umständen wie eine späte Abrechnung mit eigenen „Lebenslügen“ (Mohr 2013) an.

In diese Gruppe gehören auch die, wiederum nicht selten jüngeren, Autor*innen, deren Forschung stark mit den geschichtspolitischen Auseinandersetzungen in der deutschen Linken verbunden ist. Diese Debatten führten über eine Kritik an nationalistischen, antizionistischen und antisemitischen Elementen in der Friedensbewegung und allgemein in der Linken schließlich zur Ausbildung der sogenannten Antideutschen (vgl. Hanloser 2004; Ullrich 2008). Deren ohnehin stark akademisch sowie antipolitisch geprägter Diskurs - der gleichwohl stets auf sein Kritikobjekt, die Bewegungslinke, verwiesen bleibt - legte es wiederum nahe, dass seine Themen und Interpretationen mit einigen Protagonist*innen in das wissenschaftliche Feld diffundierten. Strukturähnlich dazu sind die Forschungen über rechtsextreme Bewegungen einzuordnen, die überwiegend von klarer Distanz bis hin zu Feindschaft der mehrheitlich wohl eher linken und liberalen
Forschenden (vgl. Gillan/Pickerill 2012: 133) gegenüber ihrem Gegenstand geprägt sind. Sie behandeln einen Teilbereich, der immer mehr die Ecke des Stiefkinds der Protestforschung verlässt, was nicht zuletzt mit der Welle völkisch grundierter Proteste zusammenhängt, die mit Pegida verbunden sind (Heim 2017).

Die Distanzierten, seien sie Renegat"innen oder Kritiker"innen bestimmter Bewegungen, haben also mit den Engagierten trotz umgekehrter Vorzeichen durchaus Gemeinsamkeiten. Die Distanzierung ist keine universelle gegenüber „Bewegung(en)“, sondern auf den Bewegungstypus der eigenen Aufmerksamkeit bezogen. Das bedeutet im Einzelfall auch, dass Forschende gleichzeitig je nach Gegenstand unterschiedliche Typen repräsentieren können.

Randbereiche und Grenzen der Typisierbarkeit Zuletzt sollen noch Akteure Erwähnung finden, die keinen homogenen Typus bilden. Gemein ist ihnen nur, dass soziale Bewegungen und Proteste zwar gelegentlich ihr Gegenstand sind, der Zugang sich aber primär durch andere thematische, theoretische oder auch politische Interessen ergibt und sich in konzeptueller wie personaler Hinsicht kaum mit dem Identitätskern der Protestforschung (dazu unten mehr) überschneidet. Dies gilt beispielsweise für die Jugendforschung. So gab es am Deutschen Jugendinstitut immer wieder gehaltvolle Forschungen zu Protesten und Protestierenden, beispielsweise eine bis heute wichtige Studie zum Verhältnis von „Demonstranten und Polizisten“ (Willems et al. 1988); allerdings gibt es dort auch inhaltlich sehr unterschiedliche angewandte Forschung im Spannungsfeld von Präventionsanstrengungen - was den Rahmen der Wissenschaft mindestens überdehnt - und kritischer Analyse unter anderem zu „Linksradikalismus/Linksextremismus“. Ähnliches wie für die Jugendforschung gilt für die Gewaltforschung und neuerdings, über die Themenfelder Radikalisierung und politischer Islam, auch für die Sicherheitsforschung. Hier kommt auch das Publikationswesen der Extremismustheoretiker"innen, etwa am Dresdener Hannah-Ahrend-Institut und Hochschulen 
des Bundes, zwischen Wissenschaft und Verfassungsschutzbehörden ins Blickfeld, deren konkrete Forschungsobjekte neben ,extremistischen' Parteien nicht zuletzt auch Bewegungen und Proteste darstellen. Auch diverse zivilgesellschaftliche Rechercheinitiativen und Archive tangieren über die immer wieder in den Blick kommende Akteursgruppe das Forschungsfeld.

All diese Bereiche würde ich zunächst nicht zur Protestforschung im engeren Sinne zählen. Diese hat eine - gewiss fragile - aber doch erkennbare Identität (Teune 2008; Rucht 2011; Teune/Ullrich 2018), die sich stark über personale Netzwerke von Forschenden und den Bezug auf ein überschaubares Set an Theorien konstituiert (vgl. Hellmann/ Koopmans 1998), welche von Marion Hamm als „mainstream of current social movement theory" zusammengefasst wurden (zit. nach Ullrich et al. 2014: 2). Diese Feldbestimmung muss ohne ein sehr starkes ableitbares Kriterium auskommen. Sie basiert auf der Selbstbezüglichkeit der Kommunikation dieses Netzwerks. Eine breitere, allein auf den Forschungsgegenstand abhebende Definition würde die hier als Randbereiche auftauchenden Forschungen einfach mit einbeziehen. Gehen wir jedoch von der gewählten Bestimmung aus, bestehen zur - wie von der Protestforschungskritik insinuiert - Sicherheitsforschung und zu Exponent"innen der Extremismustheorie nur wenig inhaltliche und personelle Überschneidungen, was aber durch Zitationsnetzwerkanalysen weiter geprüft werden müsste. Allerdings sind diese Randbereiche angesichts politischer Anreize teilweise im Wachstum begriffen und es zeichnet sich ab, dass die Übergänge, wohl vor allem zugunsten des Typs der Distanzierten, fließender werden. Dies liegt nicht zuletzt an der massiven Förderung von Sicherheitsforschung durch die EU und das BMBF und an den Förderprogrammen des Bundesfamilienministeriums, durch die außerakademische Monitoring-Einrichtungen gestärkt wurden und in einzelnen Bundesländern neue Einrichtungen geschaffen wurden, die demokratiefeindliche, rechte, militante oder ,extremistische' Bestrebungen kompetenter als der Verfassungsschutz beobachten und analysieren sollen (Teune/Ullrich 2018). Diese Forschungsstellen haben nicht nur teilweise denselben Akteurstyp im Blick, sondern sind z.T. auch an Einrichtungen angesiedelt, die zu den wenigen existenten Schwerpunkten der Protestforschung zählen (wie dem Göttinger Institut für Demokratieforschung ${ }^{7}$ ). Angesichts des expliziten politischen Präventivauftrags dieser Institutionen kommt - ob gewollt oder nicht - eine instrumentelle, versicherheitlichte Perspektive stärker denn je in die Forschung. Dies scheint bei der funktional eng mit dem Auftrag des Verfassungsschutzes verzahnten und auf dessen Daten zurückgreifenden „Forschungs- und Dokumentationsstelle zur Analyse politischer und religiöser Extremismen in Niedersachsen (FoDEx) " nur am offensichtlichsten auf (Teune/Ullrich 2018: 419), ganz sicher jedenfalls aus der Perspektive vieler linker Gruppen vor Ort (s. Endnote 3), die - dieses Kontextverständnis ist entscheidend gegenwärtig auch mit illegaler polizeilicher Datenspeicherung und V-Leuten konfrontiert sind. Unter anderen politischen Vorzeichen gilt das aber auch für die zur Beobachtung rechter Bestrebungen etablierten Einrichtungen. Der eingangs erwähnte „Gegnerkunde“-Vorwurf erscheint hier am ehesten plausibel, doch es hängt von der konkreten Arbeit der allesamt erst jüngst etablierten Einrichtungen $a b$, als wie hellsichtig oder auch nicht er sich erweisen wird, vor allem wenn er nicht mit dem Selbstbild der Kritisierten übereinstimmt (Finkbeiner/Schenke 2018). Am Ende gilt das gesprochene und vor allem geschriebene Wort.

Wie eingangs angemerkt, handelt es sich bei den vorgestellten Typen um Idealtypen, denen sicherlich einige Forschende weitgehend entsprechen. Doch in der Realität ist von großen Überschneidungen auszugehen. Diese ergeben sich aus der Vielfalt der Forschungspersönlichkeiten und deren Veränderungen im jeweiligen Lebensverlauf sowie, wie gezeigt, aus feldinternen Dynamiken und Änderungen der Rahmenbedingungen. Die möglichen Rollen von Protestforschenden für das Feld erschöpfen sich auch nicht in den Dargestellten. ${ }^{8}$ 


\section{3| Vom Nutzen der Protestforschung}

Der hier dargebotene Überblick sollte verdeutlichen, dass die Gefahr der Indienstnahme als "Gegnerkunde“ durchaus besteht, aber gerade nicht konstitutiv für die akademische Protestforschung im Allgemeinen ist, sondern zunächst abhängig von der je spezifischen Konstellation zwischen Forschenden und Forschungsfeld. Es wäre jedoch falsch, die Problematik auf die Frage von Motiven oder Haltungen zu begrenzen, sagen diese doch nur bedingt etwas über die tatsächlichen Effekte aus. Während die Kritik der Protestforschung hier in einem Negativszenario drohende Gefahren heraufbeschwört, bleibt aber die mögliche gesellschaftliche Nützlichkeit von Protestforschung weitgehend unterbelichtet. Zum Nutzen von Protestforschung möchte ich gegen diesen Reduktionismus ein wissenschaftliches, ein bewegungsnah-linkes und ein liberales Argument formulieren.

Zunächst das wissenschaftliche Argument: Der Ausdifferenzierung und steten quantitativen Ausweitung des fokussierten Partizipationstyps in der "Bewegungsgesellschaft“ (Neidhardt/Rucht 1993) entspricht ein gesellschaftliches Interesse an Aufklärung durch eine spezialisierte sozialwissenschaftliche Teildisziplin. Protestforschung als Produkt sozialer Differenzierung wäre in diesem Sinne nichts anders als technische Strömungsmechanik, Psychoonkologie oder Stellarstatistik. Hier hat sich die Forschung schon oft auch als Ausgleich gegen allzu schablonenhafte mediale Repräsentationen von Protest erwiesen. ${ }^{9}$ Dies erscheint mir nicht weiter begründungspflichtig, zumal ein Verständnis von Protest und Bewegungen einen notwendigen Beitrag $\mathrm{zu}$ anderen Forschungsfeldern leistet, die Konflikte oder sozialen Wandel behandeln.

Mit der Etablierung des Forschungszweigs geht aber auch eine Normalisierung und Rationalisierung von Protest als nun wissenschaftlich legitimierte Partizipationsform einher, weswegen Pettenkofer (2010: $85 \mathrm{ff}$.) die Protestforschung auch als „Normalisierungsunternehmen“ bezeichnet. Damit sind wir beim zweiten, dem bewegungsnahen, linken Argument für Bewegungsforschung. Denn sie hat nicht nur den allgemein an Protesten und Bewegungen Interessierten spannende Erkenntnisse zu bieten, sondern kann für Bewegungen konkret nützlich sein. Der Nutzen liegt angesichts der generell geringen Prognosekraft sozialwissenschaftlicher Theorien eher seltener als Rezeptwissen für erfolgreichen Protest vor, auch wenn man einige der Ansätze mit ihrem rationalistischen Bias durchaus als „Organisationsberatung“ lesen kann (Pettenkofer 2010: 87 ff.; vgl. a. Johnston 2009: 2; Ullrich et al. 2014), sondern überwiegend als Reflexionswissen. Bewegungsforschung wäre dann wissenssoziologische Beobachtung zweiter Ordnung (Luhmann 1997: 92-163), die den blinden Fleck in den Blick nimmt, der aus der Unmittelbarkeit des Aktivismus und aktivistischer Forschung heraus nicht analysierbar ist. In diese Kategorie gehören Arbeiten, die sich mit Spuren des Nationalismus (Ludwig 1995; Eder 2000) oder Antisemitismus (Kloke 1994; Kraushaar 2005; Ullrich 2008) in progressiven Bewegungen befassen, oder Arbeiten, die in der konsensorientierten Bewegungskultur von Basisinitiativen untersuchen, was in deren realen Praxen denn Konsens tatsächlich bedeutet - nämlich erstaunlich Unterschiedliches - oder auch verschleiert, wie man der Arbeit von Haug (2012) entnehmen kann. Gleiches gilt für Forschungen über Gegenüberwachung (also gegen staatliche Überwachung von Bewegungen gerichtete Überwachung von unten), die unintendierte und paradoxe Effekte nachwiesen, unter anderem eine reaktive Verstärkung der staatlichen Überwachung sowie Selbststigmatisierung (Wilson/Serisier 2010; Schaefer/ Steinmetz 2014; Ullrich/Knopp 2018). Gerade die Texte zu moralischen Widersprüchen innerhalb der Linken, wie Rassismus, Sexismus oder Antisemitismus in den eigenen Reihen, zeigen, dass die Grenze zwischen Renegatentum und Bereitstellung von Reflexionswissen verschwommen sein kann. Für die Möglichkeit der Nutzung dieser Literatur als Reflexionsressource ist das Produktionsmotiv auch nicht entscheidend. Genauso besteht allerdings kein 
systematischer Grund, dass Protestforschungswissen - unabhängig von den Intentionen der Forschenden - nicht auch genutzt wird, um Bewegungen besser zu kontrollieren. Die Rezeption wissenschaftlicher Arbeit ist nicht durch die Wissenschaft steuerbar.

Hier schließt mein ,liberales' Argument an: Ich gehe davon aus, dass eine solche Indienstnahme faktisch selten erfolgt. Vielmehr ist anzunehmen, dass soziale Differenzierung dies weitgehend verhindert. Dafür sorgen die jeweiligen feldspezifischen Habitus und Sprachspiele oder Codes, die nur über eine geringe Anschlussfähigkeit über Feldgrenzen hinweg verfügen. Die Mehrheit der wissenschaftlichen Texte ist für Sicherheitsbehörden nicht anschlussfähig; gleiches gilt wahrscheinlich für viele Protestakteure. Stattdessen wird die mühselige Übersetzungsnotwendigkeit von Wissenschaft für die Praxis beispielsweise aus der Polizei richtiggehend beklagt (Weitemeier 2002). Doch auch diese These bedarf einer empirischen Prüfung.

\section{4| Ambivalenzen des Feldes und ethische Imperative ${ }^{10}$}

Selbst wenn wir die hier explorativ entwickelten Annahmen als richtig unterstellen, wird die so herausgearbeitete weitgehende ethische „Unbedenklichkeit“ von Protestforschung per se begrenzt durch bestimmte offensichtliche Risiken, insbesondere für die Beforschten.

Damit sind wir wiederum bei dem Rest der protestforschungsfeindlichen Anwürfe, dem aus Sicht der Forschungsethik, welche ja vor allem mögliche Nachteile von Forschung für die Beforschten reflektiert, nicht ohne Weiteres widersprochen werden kann. Trotz aller dargelegten Erwägungen ist eine Verwendung von Forschungsergebnissen zur Kontrolle, Kooptation oder gar Repression von Bewegungen möglich (oder, wie oben geschildert, manchmal sogar gewünscht). Die Risiken betreffen insbesondere vulnerable Bewegungen, die Repression ausgesetzt sind oder über geringe Beschwerdemacht verfügen. Hier sind außer Absprachen im Forschungsbündnis kontinu- ierliche Reflexionen darüber angezeigt, wie publiziertes Wissen den Beforschten schaden könnte und gegebenenfalls sind Informationen nicht zu verwerten, wenn auch ausgefeilte Anonymisierung nicht für ausreichenden Schutz zu sorgen verspricht.

Ein besonderes Einfallstor für ethische Probleme sind auch aktuelle Entwicklungen im wissenschaftlichen Feld, insbesondere die steigende Konkurrenz um Forschungsmittel im akademischen Kapitalismus (Münch 2011) und die als Ausgleichsmöglichkeit sich anbietenden ,unmoralischen ' Angebote aus dem Bereich der angewandten und auf Prävention zielenden Forschung. Sie verschärfen die durch allgemeine Konkurrenz um Reputation und Ressourcen ohnehin kontinuierlich bestehenden Anreize zu unethischem Verhalten, wie Ausnutzung der Beforschten oder Intransparenz über Auftraggeber, um den Feldzugang zu verbessern. Auch der "Seitenwechsel“ zu Sicherheitsorganen kann eine individuell naheliegende Option sein, um dem akademischen Dauerprekarität zu entkommen, die zudem im Feld Protestforschung angesichts seiner schlechten Institutionalisierung besonders virulent ist. Das hier angesprochene Problem des Rollenübergangs tritt jedoch schon unterhalb dieser Schwelle auf, da es Einflussnahmen subtiler bis offensiver Natur durch Fördergeber, Kooperationspartner und andere gibt, welche man in den Verstrickungen der Praxis wohl oft nicht nur unter Wahrung allerhöchster Ethikideale wählen kann. Gerade in Kooperationen im Bereich der Sicherheitsforschung ist es nicht leicht zu entscheiden, ob die eigene Perspektive eher wirksamer kritischer Impuls oder eher sozialwissenschaftliches Feigenblatt für repressive Sozialtechnologie ist. Alle allgemeingültigen forschungsethischen Fragen tauchen also auch in der Protestforschung auf, werden aber durch einige Besonderheiten des Gegenstandes verstärkt. Daher rühren auch die ,verstärkten' forschungsethischen Imperative nach kontinuierlicher Reflexion der Strukturen des wissenschaftlichen Feldes und ihres Wandels hinsichtlich seiner möglichen Einflüsse auf die Forschung; größtmöglicher Transparenz 
über Sprechort, Auftrag und Finanzierung; strengstem Datenschutz und gegebenenfalls Nichtverwendung von Daten; der Stärkung theoretischer und methodologischer Debatten als Gegengewicht gegen politisch kontingente Positionierungen und kontinuierlicher Reflexion der (wissenschaftlichen, politischen, ethischen usw.) Grundlagen der eigenen Forschung in einem auf Dauer angelegten Meta-Diskurs über das eigene Fach (Teune/Ullrich 2018).

\section{5| Fazit: Protestforschung als soziologi- sche Aufklärung}

Die Widersprüchlichkeit der Anrufungen, in der man sich als Protestforscher" in bewegt, ist nicht grundsätzlich aufzulösen, sondern verlangt ein bewusstes Agieren im Wissen um die Ambivalenzen und in ihrer aktiven Annahme, mithin einen Platz „Zwischen den Stühlen “11. Sinnvoll, richtig und ethisch unbedenklich erscheint mir eine transparente, selbstreflexive Protestforschung als soziologische Aufklärung, die sich der eigenen Voraussetzungen nie zu selbstgefällig-sicher sein darf, um ein Abgleiten in das Ausruhen auf etablierten Praxen oder das unmoralische Ausnutzen ebenso zu verhindern wie eine reine (erkenntnisabträgliche) Servicehaltung für Bewegungen.

Trotz bestehender Risiken für Beforschte folgt aus der Kombination der vorgebrachten Argumente für mich - als Forschender und als politischer Mensch, der vielen progressiven Bewegungen positiv gegenübersteht - dass die Schaffung von Wissen, dessen Verwendung durch die Produzent"innen nicht steuerbar ist, der aktiven Selbstverdummung durch das Vermeiden von Fragen vorzuziehen ist. Definitiv keine gute Antwort ist die von radikalen Kritiker*innen immer wieder nahegelegte Option der schlichten Negierung der Möglichkeit oder Sinnhaftigkeit von Protestforschung, quasi ein Frageverbot oder zumindest -verzicht, weil Ergebnisse sich als ,nicht nützlich` erweisen könnten. Die fatalen und inakzeptablen Folgen eines solchen Ansatzes sollten beispielsweise aus dem dogmatischen Marxismus-Leninismus reichlich bekannt sein.
Peter Ullrich, Dr. phil. Dr. rer. med., ist Ko-Leiter des Forschungsbereichs „Soziale Bewegungen, Technik, Konflikte" am Zentrum Technik und Gesellschaft sowie Fellow am Zentrum für Antisemitismusforschung der TU Berlin, außerdem Mitglied des Instituts für Protest- und Bewegungsforschung, http:// textrecycling.wordpress.com, ullrich@ztg. tu-berlin.de

\section{Literatur}

Baumgarten, Britta/Rucht, Dieter 2013: Die Protestierenden gegen ,Stuttgart 21 ${ }^{\circ}$ - einzigartig oder typisch? In: Brettschneider, Frank/ Schuster, Wolfgang (Hg.): Stuttgart 21. Ein Großprojekt zwischen Protest und Akzeptanz. Wiesbaden: Springer VS, 97-125.

Bourdieu, Pierre 1993: Über einige Eigenschaften von Feldern. In: Soziologische Fragen, 5. Aufl. Frankfurt am Main: Suhrkamp Verlag.

Bourdieu, Pierre 1998: Vom Gebrauch der Wissenschaft. Für eine klinische Soziologie des wissenschaftlichen Feldes. In: Egger, Stephan (Hg.): Édition discours 12. Konstanz: UVK, Univ.-Verl.

Daphi, Priska/Rucht, Dieter/Stuppert, Wolfgang/Teune, Simon/Ullrich, Peter 2014: Occupy Frieden. Eine Befragung von Teilnehmer/innen der „Montagsmahnwachen für den Frieden“. ipb working papers. Berlin. https:// protestinstitut.files.wordpress.com/2015/03/ occupy-frieden_ipb-working-paper_web.pdf. [30.11.2018]

Eder, Klaus 2000: Kulturelle Identität zwischen Tradition und Utopie. Soziale Bewegungen als Ort gesellschaftlicher Lernprozesse. Frankfurt am Main/New York: Campus.

Finkbeiner, Florian/Schenke, Julian 2018: Der Aktivist als ,besserer' Forscher? Göttinger Antwort auf Berliner Kritik. In: Forschungsjournal Soziale Bewegungen, Jg. 31, Heft 3, 93-97.

Gillan, Kevin/Pickerill, Jenny 2012: The Difficult and Hopeful Ethics of Research on, and with, Social Movements. In: Social Movement Studies, Jg. 11, Heft 2, 133-143.

Hanloser, Gerhard (Hg) 2004: „Sie warn die Antideutschesten der deutschen Linken“. Zu 
Geschichte, Kritik und Zukunft antideutscher Politik. Münster: Unrast.

Haug, Christoph 2012: Assembly Publics and the Problem of Hegemony in consensus Decision-making. http://www.opendemocracy. net/christoph-haug/assembly-publics-and-problem-of-hegemony-in-consensus-decision-making. [18.10.2013]

Haunss, Sebastian/Ullrich, Peter 2013: Viel Bewegung - wenig Forschung. $\mathrm{Zu}$ - und $\mathrm{Ge}-$ genstand sozialwissenschaftlicher Protest- und Bewegungsforschung in der Bundesrepublik. In: Soziologie, Jg. 42, Heft 3, 290-304.

Heim, Tino (Hg.) 2017: Pegida als Spiegel und Projektionsfläche. Wechselwirkungen und Abgrenzungen zwischen Pegida, Politik, Medien, Zivilgesellschaft und Sozialwissenschaften. Wiesbaden: Springer VS.

Heinelt, Peer 2017: Repressive Wissenschaft. Gegnerkunde. Wie sich akademische Einrichtungen mit Untersuchungen über abweichendes Verhalten zu Gehilfen des Staatsschutzes machen. In: junge welt, 5. April 2017, Abschn. 105.

Hellmann, Kai-Uwe/Koopmans, Ruud 1998: Paradigmen der Bewegungsforschung. Entstehung und Entwicklung von Neuen Sozialen Bewegungen und Rechtsextremismus. Wiesbaden: VS Verlag für Sozialwissenschaften. Johnston, Hank (Hg.) 2009: Culture, Social Movements, and Protest. Aldershot: Ashgate.

Klein, Ansgar/Hellmann, Kai-Uwe (Hg.) 1998: Bewegungen von Rechts? Analyse und Kritik. In: Forschungsjournal Neue Soziale Bewegung, Jg. 1994, Heft 1, 2-13.

Kloke, Martin 1994: Israel und die deutsche Linke. Zur Geschichte eines schwierigen Verhältnisses. Frankfurt am Main: Haag + Herchen.

Konsortium Bundesbericht Wissenschaftlicher Nachwuchs (Hg.) 2017: Bundesbericht Wissenschaftlicher Nachwuchs 2017. Statistische Daten und Forschungsbefunde zu Promovierenden und Promovierten in Deutschland. Bielefeld: W. Bertelsmann.

Kraushaar, Wolfgang 2005: Die Bombe im Jüdischen Gemeindehaus. Hamburg: Hamburger Edition.
Leinius, Johanna/Vey, Judith/Hagemann, Ingmar 2017: Poststruktualistische Perspektiven auf soziale Bewegungen. Plädoyer für eine notwendige Blickverschiebung. In: Forschungsjournal Soziale Bewegungen, Jg. 30, Heft 4, 6-19.

Leistner, Alexander 2017: Gewalt als soziale Situation. Formen und Folgen am Beispiel einer Ultragruppierung. In: Grau, Andreas/ von der Heyde, Judith/Kotthaus, Jochem/ Schmidt, Holger/Winands, Martin (Hg.): Sozialwissenschaftliche Perspektiven der Fußballfanforschung. Weinheim: Beltz Juventa, 114-30.

Ludwig, Andrea 1995: Neue oder deutsche Linke? Nation und Nationalismus im Denken von Linken und Grünen. Nation und Nationalismus im Denken von Linken und Grünen. Opladen: Westdeutscher Verlag.

Luhmann, Niklas 1997: Die Kunst der Gesellschaft. Frankfurt am Main: Suhrkamp.

Malthaner, Stefan/Teune, Simon/Ullrich, Peter 2018: Eskalation. Dynamiken der Gewalt im Kontext der G20-Proteste in Hamburg 2017. Berlin: Institut für Protest- und Bewegungsforschung. https://g20.protestinstitut. eu/wp-content/uploads/2018/09/Eskalation_Hamburg2017.pdf. [6.9.2018]

Mohr, Markus 2013: Irgendwie durchgerutscht. Die Lebenslügen des Wolfgang Kraushaar. Streifzüge Online. http://www. streifzuege.org/2013/irgendwie-durchgerutscht [4.12.2013].

Mohr, Markus/Rübner, Hartmut 2010: Gegnerbestimmung. Sozialwissenschaft im Dienst der innereren Sicherheit. Münster: Unrast.

Münch, Richard 2011: Akademischer Kapitalismus. Zur politischen Ökonomie der Hochschulreform. Berlin: Suhrkamp.

Neidhardt, Friedhelm/Rucht, Dieter 1993: Auf dem Weg in die ,Bewegungsgesellschaft'? Über die Stabilisierbarkeit sozialer Bewegungen. In: Soziale Welt, Heft 44, 305-326.

Pettenkofer, Andreas 2010: Radikaler Protest. Zur soziologischen Theorie politischer Bewegungen. Frankfurt am Main: Campus.

Roose, Jochen/Dietz, Hella (Hg.) 2016: Social Theorie and Social Movements. Mutual 
Inspirations. Wiesbaden: Springer Fachmedien Wiesbaden.

Rucht, Dieter 2011: Zum Stand der Forschung zu sozialen Bewegungen. In: Forschungsjournal Soziale Bewegungen, Jg. 24, Heft 3, 21-47.

Schaefer, Brian P./Steinmetz, Kevin F. 2014: Watching the Watchers and McLuhan's Tetrad: The Limits of Cop-Watching in the Internet Age. In: Surveillance \& Society, Jg. 12, Heft 4, 502-515.

Scholl, Armin 2019: Das Selbstverständnis der Bewegungsforschung im Dilemma zwischen Selbst- und Fremdbestimmung. Versuch einer Weiterführung der Debatte. Soziologieblog. https://soziologieblog.hypotheses. org/12318 (14.1.2019).

Teune, Simon 2008: , Gibt es so etwas überhaupt noch?' Forschung zu Protest und soziale Bewegungen. In: Politische Vierteljahresschrift, Jg. 49, Heft 3, 528-547.

Teune, Simon/Ullrich, Peter 2018: Protestforschung mit politischem Auftrag? In: Forschungsjournal Soziale Bewegungen, Jg. 31, Heft 1, 418-425.

The Autonomous Geographies Collective 2010: Beyond Scholar Activism: Making Strategic Interventions Inside and Outside the Neoliberal University. In: ACME: An International E-Journal for Critical Geographies, Jg. 9, Heft 2, 245-275.

Ullrich, Peter 2008: Die Linke, Israel und Palästina. Nahostdiskurse in Großbritannien und Deutschland. Berlin: Dietz.

Ullrich, Peter/Daphi, Priska/Baumgarten, Britta 2014: Protest and Culture. Concepts and Approaches in Social Movement Research. An Introduction. In: Baumgarten, Britta/Daphi, Priska/Ullrich, Peter (Hg.): Conceptualizing Culture in Social Movement Research. Basingstoke: Palgrave Macmillan, 1-20.

Ullrich, Peter/Knopp, Philipp 2018: Protesters' Reactions to Video Surveillance of Demonstrations. Counter-Moves, Security Cultures, and the Spiral of Surveillance and Counter-Surveillance. In: Surveillance \& Society, Jg. 16, Heft 2, 183-202.

Vey, Judith 2015: Gegen-hegemoniale
Perspektiven. Analyse linker Krisenproteste in Deutschland 2009/2010. Hamburg: VSA.

Weitemeier, Ingmar 2002: Anforderungen und Erwartungen an die wissenschaftliche Forschung aus Sicht der Polizei. In: Bornewasser, Michael (Hg.): Empirische Polizeiforschung III. Herbolzheim: Centaurus Verlag \& Media, 3-12. Willems, Helmut/Eckert, Roland/Goldbach, Harlad/Loosen, Toni 1988: Demonstranten und Polizisten. Motive, Erfahrungen und Eskalationsbedingungen. DJI Forschungsbericht. München: Verlag Deutsches Jugendinstitut e. V./Juventa.

Wilson, Dean Jonathon/Serisier, Tanya 2010: Video Activism and the Ambiguities of Counter-Surveillance. In: Surveillance \& Society, Jg. 8, Heft 2, 166-180.

\section{Anmerkungen}

${ }^{1}$ Ich danke Judith Vey für Anregungen zur Strukturierung des Feldes sowie Simon Teune, Dieter Rucht und den Herausgeber"innen für Hinweise und kritische Kommentare.

2 http://akkffm.blogsport.de/2016/11/08/ achtung-exzellenzcluster-will-linke-strukturen-ausforschen/ (10.11.2018)

3 Vgl. bspw. das Hausverbot für IfD-Mitarbeiter*innen durch linke Göttinger Gruppen (https://de.indymedia.org/node/24603, 21.11.2018) sowie die Diskussion zwischen Mitgliedern des ipb und des IfD (Teune/Ullrich 2018; Finkbeiner/Schenke 2018; Scholl 2019).

${ }^{4}$ Dass diese Disziplin, die „Protest- und Bewegungsforschung“, zwischen verschiedenen Fächern steht, insbesondere der Soziologie, der Politikwissenschaft, aber auch der Medien- und Geschichtswissenschaft, fügt ihr einige Besonderheiten hinzu, beispielsweise die Etablierung eines eigenen, begrenzten Theoriekanons, der nur in loser Beziehung zu den Grundlagentheorien der „Mutterfächer“ steht (vgl. Roose/Dietz 2016).

${ }^{5}$ Sie mit diesem Thema fortzusetzen ist angesichts der schwachen Institutionalisierung der Protestforschung in Deutschland schwieriger (Rucht 2011; Haunss/Ullrich 2013). 
${ }^{6}$ Das betrifft, wie geschildert, beide Seiten der Wissenschaft, nicht nur die in der illusio des Feldes betonte Suche nach Erkenntnis und Wahrheit, sondern auch die administrative oder hochschulpolitische Seite von Wissenschaft als Lohnarbeit sowie Arena von Kämpfen um Status, Reputation und Ressourcen (Bourdieu 1993, 1998).

7 „Bundesfachstelle linke Militanz“ und „Forschungs- und Dokumentationsstelle zur Analyse politischer und religiöser Extremismen in Niedersachsen (FoDEx)", die in einem eigenartigen Spannungsverhältnis zur sicherheitspolitischen Beauftragung stehen, $\mathrm{da}$ die Beteiligten sich in bisherigen Publikationen einerseits kritisch mit dem Extremismusparadigma auseinandersetzen, es jedoch andererseits gleichzeitig aktiv benutzen und damit stabilisieren.

${ }^{8}$ Allein ein schneller Blick auf die „Schlüsselfiguren Sozialer Bewegungen“ von Leistner
(2017) zeigt, dass Protestforscher*innen dank ihres spezifischen Hintergrundes mehrere dieser Rollen gut ausfüllen können oder dies realiter tun.

${ }^{9}$ Man denke beispielsweise an die empirische Relativierung zentraler Annahmen über angeblich konservative Wutbürger (Baumgarten/Rucht 2013) oder an das, gemessen an den politisch-medialen Einschätzungen, äußert differenzierte und widersprüchliche Bild der Montagsmahnwachenbewegung 2014/15 (Daphi et al. 2014) oder der Proteste gegen den G20-Gipfel in Hamburg (Malthaner et al. 2018).

${ }^{10} \mathrm{Vgl}$. auch die Ausgabe 2/2012 von Social Movement Studies mit dem Schwerpunktthema "The Ethics of Research on Activism".

${ }^{11}$ So Finkbeiner und Schenke (2018) - ein Zitat, das allerdings auch verdeutlicht, dass zwischen den Stühlen sehr viel Platz ist. 